\title{
Gas-Surface Interactions in Low-Earth Orbit
}

\author{
Kenneth Moe and Mildred M. Moe
}

Space Environment Technologies, 23 Purple Sage, Irvine, CA 92603, USA

\begin{abstract}
When the space age began, some aerodynamicists expected that the surfaces of spacecraft would be cleaned by desorption in the high vacuum of space; while others, familiar with experiments on engineering surfaces, believed that satellite surfaces would be contaminated. During subsequent decades, satellite evidence has accumulated, showing that surfaces in low-Earth orbit are contaminated by adsorbed atomic oxygen and its reaction products. These contaminants cause accommodation coefficients to be high, and the angular distribution of reemitted molecules to be nearly diffuse. These surface conditions must be considered in calculating satellite drag coefficients in free-molecular flow. We describe the experimental and theoretical developments which have led to these conclusions.
\end{abstract}

Keywords: Rarefied gas dynamics, Satellite drag coefficients, Adsorption, Atomic oxygen, Free-molecular flow, LowEarth orbit. PACS: 94.05.Hk, 47.45.Dt, 34.35. +a, 68.43.-h, 34.50.Lf

\section{INTRODUCTION: EARLY LABORATORY MEASUREMENTS}

During the First World War, Irving Langmuir [1] demonstrated that ordinary surfaces are not clean, but are coated with gases and liquids. He improved the methods of cleaning surfaces, and developed a model of adsorption. Then in the 1930s, J. K. Roberts [2] improved Langmuir's methods of cleaning surfaces, and made the first measurements of energy accommodation coefficients on truly clean surfaces. The energy accommodation coefficient (AC) is defined as the ratio (Ei- Er) / (Ei-Ew). Here Ei is the kinetic energy of the incoming particles relative to the surface, Er is the kinetic energy of those reemitted from the surface after collision, and Ew is the kinetic energy the reemitted particles would have if they left the surface at the temperature of the surface (wall).

Roberts showed that freshly cleaned surfaces have low accommodation coefficients, but that they rise to high values during several hours, as contaminants collect on the surfaces, even in a high-vacuum system. After the Second World War, research on energy accommodation on clean and dirty surfaces flourished [3,4]: The typical laboratory experiment showed that incident molecules are reflected from contaminated surfaces with a high AC in a diffuse angular distribution. With sufficient effort, experimenters could produce clean surfaces. On such surfaces, the ACs usually were much lower and the angular distributions quasispecular in most cases. (Only a few of these measurements were made with incident energies of 2 to $10 \mathrm{eV}$, which are typical of satellite collisions with air molecules in low-Earth orbit.)

Lloyd Thomas, the dean of American molecular-beam experimenters, writing in [4], showed that various highvacuum experimenters had measured ACs of gases on tungsten ranging from 0.02 to 1.0 . He attributed much of this variation to differences in the quantity and species of the contaminants in the various vacuum systems. Thomas, his students, and numerous colleagues performed many experiments that showed the dependence of AC on surface material, surface contamination, surface temperature, and the mass of the incoming molecule [3, 4]. (Spacecraft temperatures are kept near $300 \mathrm{~K}$ so that the electronics inside will function properly.) Busby and Trilling commented in [3] that the accommodation coefficient of a gas on a particular clean surface is approximately half of that of the same gas on the contaminated surface.

In 1981, Thomas summarized 40 years of laboratory measurements of accommodation at the University of Missouri [5]. Experimental laboratory measurements continued to accumulate, and in 1990, Brundle and Broughton published 350 pages in [6] on the interaction of oxygen with clean metal surfaces. We have learned much about adsorption and energy accommodation from laboratory measurements. However, we cannot apply laboratory measurements directly to surfaces in space because the AC depends on the amount of atomic oxygen adsorbed on the surface as well as other factors: Atomic oxygen, a principal constituent of the thermosphere, adsorbs on nearly 
any surface [7, 8], and specifically on spacecraft materials [9-17]. Oxygen also reacts chemically with many spaceborne materials [12-16].

\section{MEASUREMENTS IN SPACE}

When the space age began, rocket and satellite measurements showed how gas-surface interactions occur on surfaces and within instruments in space: Atmospheric measurements by rocket-borne pressure gauges disagreed with theory, so the pressure gauges were spun to separate outgassing from the density measurement [18]. A similar difficulty was encountered when pressure gauges were flown on satellites [19]. The densities measured by the gauge disagreed with those derived from orbital drag observations. Outgassing should have ended after a few days in orbit. The continued disagreement was finally resolved by adding terms representing adsorption and desorption to the differential equation governing the density measurement [9-11]. The theoretical pressure history without adsorption for one spin cycle of the Redhead gauge on Explorer 17 is compared with the measured pressure in Fig. 1a. The influx, efflux, desorption, and adsorption in the gauge are shown in Fig. 1b, along with their time integral, which is the pressure history. The pressure history which would have been measured if there had been no adsorption or desorption is also shown dashed. Fig. 1 demonstrates that adsorption and desorption occur in space-borne instruments.
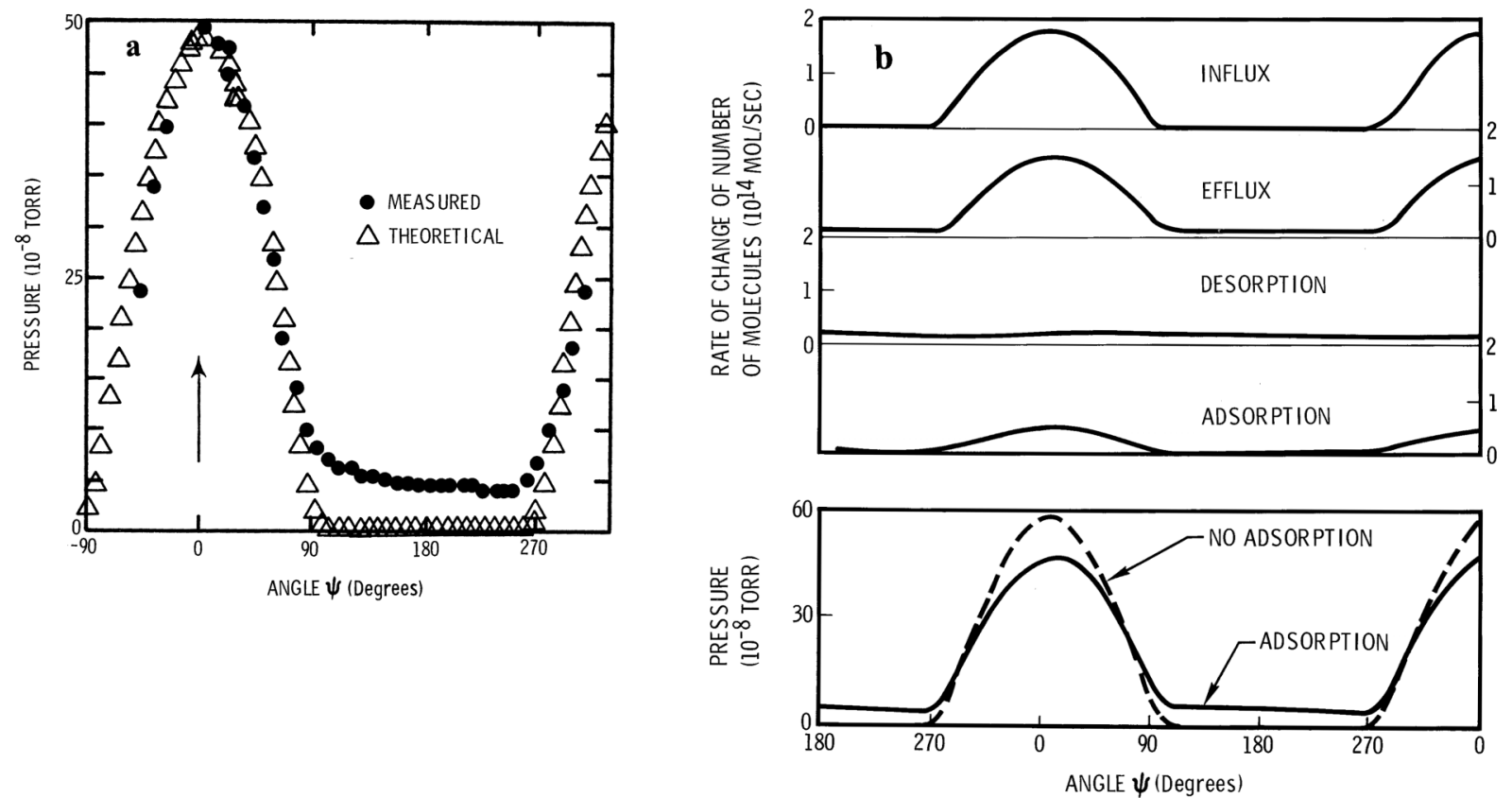

FIGURE 1A, 1B. The pressure history during one spin cycle of the Redhead gauge on Explorer 17 (after Newton, et al.) is shown in 1a. The physical processes contributing to the gauge pressure are shown in Fig. 1b. The time integral of these processes is the pressure at the bottom (solid line). The dashed line is the pressure history that would have transpired if there had been no adsorption.

Much of the adsorption measured in orbit involves atomic oxygen. Hedin, et al. [12] showed in Fig. 2 how the surface coverage of atomic oxygen (adsorbed on the interior walls of the mass spectrometer on OGO-6) varied as the satellite flew down to perigee, then back up to apogee. At its perigee of $400 \mathrm{~km}$, about $5 \%$ of a monolayer of atomic oxygen was adsorbed on the interior walls of the mass spectrometer. This agrees with laboratory experiments which have shown that atomic oxygen adsorbs on many surfaces $[7,8,17]$. The realization that atomic oxygen reacts chemically within spaceborne instruments has led to improvements in measurements of upper atmospheric composition [20]. 


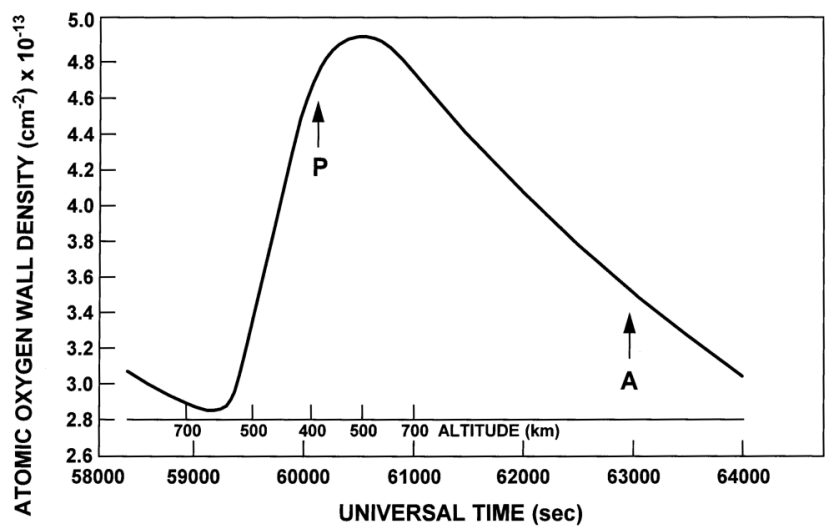

FIGURE 2. Atomic oxygen adsorbed in the mass spectrometer on OGO-6 during one orbital revolution (after Hedin, et al.). Perigee is point $\mathrm{P}$, and apogee is point $\mathrm{A}$.

In the meanwhile, Gregory and Peters [14] measured the angular distribution of oxygen atoms scattered off a carbon surface at $225 \mathrm{~km}$ on Space Shuttle flight STS-8. They interpreted the measurements to show "a high degree of accommodation characterized by (angular) distributions approaching the cosine". This is different from laboratory measurements on clean surfaces, which usually observe lower accommodation and quasispecular angular distributions.

The conclusions of Gregory and Peters' Space Shuttle experiment are further supported by the existence of "spacecraft glow" which appears on the forward facing surfaces of spacecraft. Much of the energy of collision is dissipated in the form of optical radiation from excited molecules on the spacecraft surface, and from other excited molecules as they flow away from the surface. Yee et al. [21] demonstrated that the energy emitted optically is of the same order of magnitude as the energy of collision.

\section{DRAG COEFFICIENTS}

When satellites began to fly, scientists had the opportunity to deduce atmospheric densities from the observed orbital decay. To achieve that goal, they had to know the characteristics of gas-surface interactions in orbit to determine the appropriate drag coefficient. Some scientists believed that satellite surfaces were cleaned by desorption caused by the low air density, while others believed that satellite surfaces were contaminated like most engineering surfaces. The gas-surface interaction model of Sentman [22] assumed that the reemission is completely diffuse, so it was based on the assumption that satellite surfaces are contaminated. The gas-surface interaction model of Schamberg was more general, allowing for reemission covering the full range from diffuse to quasispecular [23, 24]. Both models are still employed by some analysts to calculate drag coefficients.

At the beginning of the space age, many different drag coefficients were used by scientists who wanted to deduce atmospheric density from orbital decay [25], which provides only relative densities. These workers often adopted a fixed value of drag coefficient, such as 2.0 or 2.2. This was a sensible procedure because neither drag coefficients nor accommodation coefficients had been measured in orbit at that time. Drag coefficients were uncertain by $50 \%$, while thermospheric air densities varied with latitude, longitude, altitude, and solar activity by several orders of magnitude. In 1965 and 1966, both M. N. Izakov [26] and Graham Cook [27, 28] studied laboratory measurements of energy accommodation, and concluded that 2.2 would be a good average number to adopt as the standard drag coefficient. After Desmond King-Hele and Luigi Jacchia accepted this value, it was generally adopted by people who worked with orbital decay and accelerometer measurements of density. A constant drag coefficient of 2.2 simplified the development of thermospheric models. It is still widely used (particularly for spacecraft of compact shapes).

In the late 1960s a new method of separating the air density from the drag coefficient became available, with the analysis of the spin decay and orbital decay of paddlewheel satellites [29-32]. This method made use of the unpublished theoretical analysis of the spin decay of the first paddlewheel satellite, Explorer 6, which was performed by Leon and Reiter in 1959, before the satellite was launched. The analysis and the flight results were presented by Reiter and Moe at the 6th Rarefied Gas Dynamics Symposium [3] and in [29]. These were the first measurements of absolute thermospheric densities. An independent analysis of the Explorer 6 measurements by 
Gerald Karr confirmed the usefulness of paddlewheel satellite measurements [30]. Karr used a model of gas-surface interaction that he himself had developed. Analyses [31,32] for two more paddlewheel satellites soon followed. As attitude control systems improved, paddlewheel satellites fell out of favor, so this source of information dried up; however Ching, et al. [33] used the lift and drag of the S3-1 satellite to measure accommodation coefficients at a perigee altitude of $169 \mathrm{~km}$ in low-Earth orbit, and confirmed Beletsky's high value of accommodation coefficient below $200 \mathrm{~km}$ [32]. Imbro, et al. [34] later analyzed more Ariel 2 data, and confirmed the analysis in [31]. Table 1 lists the energy accommodation coefficients deduced from these four early satellites, three in low-Earth orbits, and one (Explorer 6) in a geosynchronous-transfer orbit.

TABLE 1. Accommodation Coefficients Measured by Four Early Satellites

\begin{tabular}{lcccc}
\hline Satellite & Perigee, $\mathbf{k m}$ & Orbital eccentricity & $\begin{array}{c}\text { Accommodation } \\
\text { coefficient }\end{array}$ & Source \\
\hline S3-1 & 159 & 0.22 & $0.99-1.00$ & Ching et al. (1977) \\
Proton 2 & 168 & 0.03 & 1.00 & Beletsky (1970) \\
Ariel 2 & 290 & 0.07 & 0.86 & Imbro et al. (1975) \\
Explorer 6 & 260 & 0.76 & 0.65 & Moe (1966) \\
Explorer 6 & 260 & 0.76 & 0.74 & Karr (1969) \\
\hline
\end{tabular}

Calculations based on models of drag coefficient and on experiments with long, attitude-controlled satellites demonstrated that satellites of different shapes have differing drag coefficients [35-37]. This had already been observed when long cylindrical reconnaissance satellites flew in the 1960s. Unfortunately, the reconnaissance satellites were classified, so these results were unknown to the general scientific community until the data were declassified in 1971 [38], and little publicized thereafter. The comparison of the reconnaissance satellite data with density models based on measurements by satellites of compact shapes also showed that Sentman's drag coefficient model was accurate near $200 \mathrm{~km}$ [39]. This suggested that reemission is diffuse at this altitude. In the same paper [39], an alternative calculation used Schamberg's model to calculate drag coefficients assuming a quasispecular angular distribution of reflected molecules near $200 \mathrm{~km}$. This calculation produced a $70 \%$ disagreement between modeled drag and measured drag of the long cylindrical satellites. We conclude that reemission is diffuse near 200 $\mathrm{km}$ altitude, even near grazing incidence on the sides of long cylindrical satellites which fly like an arrow [39].

In the laboratory measurements on clean surfaces, Trilling [p. 410 in 4] and Thomas in [40] showed that the AC usually depends strongly on the surface material. In contrast, laboratory experiments had shown that the accommodation coefficients of various gases on contaminated metal surfaces are almost independent of the metal used $[41,42]$. To see whether the same thing held true in space, we studied NASA's ODERACS experiment, which flew half a dozen spheres with different surface materials and surface treatments [43]. The result was that the average drag coefficients of pairs of rough aluminum and smooth chrome spheres differed by only $1 \%$ at $200 \mathrm{~km}$, and $4 \%$ at $340 \mathrm{~km} \mathrm{[44].} \mathrm{Tan} \mathrm{and} \mathrm{Badhwar} \mathrm{had} \mathrm{also} \mathrm{found} \mathrm{similar} \mathrm{results,} \mathrm{using} \mathrm{the} \mathrm{first} \mathrm{month} \mathrm{of} \mathrm{ODERACS} \mathrm{data}$ [45]. We concluded that the drag coefficients (and accommodation coefficients) of satellites in low-Earth orbit are nearly independent of the substrate material. We believe that this insensitivity is caused by the adsorption of atomic oxygen and its reaction products on the satellites' exterior surfaces, which shields the surfaces from direct contact. Van der Waals forces extend the influence of the adsorbed molecules out to several molecular diameters [F. P. Bowden in Reference 4].

The initial perigee height of the ODERACS spheres was only about $340 \mathrm{~km}$, so there was the question of whether satellites at much higher altitudes would have enough surface contamination to cause diffuse reemission. Further study [44] utilizing Calspheres 3, 4, and 5 (launched together into a circular orbit at $775 \mathrm{~km}$ ) led to the conclusion that the lifetimes were consistent with the assumption of diffuse reemission but inconsistent with the assumption of quasispecular reemission. (Pardini et al. [46] later measured the drag coefficients of all three Calspheres at $500 \mathrm{~km}$ to be 2.31 when diffuse reemission was assumed.)

The fact that reemission is diffuse, even at grazing incidence in low-Earth orbit, reduces the uncertainty in planning the CLIPPER [47] and SLATS [48] spacecraft experiments. If these spacecraft are flown near solar maximum, the accommodation coefficients measured by Pardini, et al. [46] will be appropriate. If flown at times of lower solar activity, the accommodation coefficients provided in [37] can be used. For several simple shapes, the drag coefficients appropriate to accommodation coefficients from 1.00 to 0.90 are provided in [37].

It is important to emphasize that the accommodation coefficients in low-Earth orbit given in Table 1 were measured at times of low solar activity. Therefore, they do not apply near sunspot maximum, when the 
thermosphere contains more atomic oxygen. Pardini et al. [46] recently have measured accommodation coefficients near sunspot maximum, which are indeed higher than those in Table 1. Pilinski et al. [49] are using the Langmuir adsorption isotherm to solve for the relationship between AC and atomic oxygen concentration. Such improvements in our understanding of gas-surface interactions in orbit could aid the efforts to validate the UV spectrometric sensors on the new DMSP satellites [50]; as well as the efforts to refine the density measurements by the precise new accelerometers on the CHAMP and GRACE satellites [51-53]. All of this work could then contribute to the long-term program to improve the measuring and modeling of the thermosphere [54, 55].

\section{CONCLUSIONS}

Atomic oxygen, a principal constituent of the thermosphere, adsorbs on satellite surfaces in low-Earth orbit, and causes the incident air molecules to be diffusely reemitted with high energy accommodation. For this reason, satellite drag coefficients should be calculated from energy accommodation coefficients measured in orbit, rather than from those measured in laboratory experiments, which cannot duplicate orbital conditions. This quest for realistic energy accommodation coefficients and the consequent improvements in satellite drag coefficients will contribute to the measurement and modeling of the Earth's high atmosphere.

\section{ACKNOWLEDGMENTS}

We want to thank Marcin Pilinski for sending us his papers before publication. We thank George Newton for Fig. 1a and Alan Hedin for Fig. 2. We also thank Karen A. Olds for help with the references.

\section{REFERENCES}

1. Langmuir, I., "The Evaporation, Condensation, and Reflection of Molecules, and the Mechanism of Adsorption," part 1, Physical Review, 8, 1916, 149-176, and many subsequent papers.

2. Roberts, J. K., "Exchange of Energy Between Gas Atoms and Solid Surfaces," Proceedings of the Royal Society, Series A, vol. 129, Sept. 1930, pp. 146-161; Pt. 2, vol. 135, Feb. 1932, pp. 192-205; and Pt. 3, vol. 142, Nov. 1933, pp. $518-524$.

3. Trilling, L. and Wachman, H. Y., "Rarefied Gas Dynamics, Proceedings of the Sixth Symposium," Vol. 2, Academic Press, NY, pp. 1015-1501, and London, (1969), and many other proceedings in the series.

4. Saltsburg, H., Smith, Jr., 'J. N., and Rogers, M. (Eds.), "Fundamentals of Gas-Surface Interactions," Academic Press, N. Y. 1967, pp. 346-391, 406-414, and 448-521.

5. Thomas, L. B., "Accommodation of Molecules on Controlled Surfaces - Experimental Developments at University of Missouri, 1940-1980”, Rarefied Gas Dynamics, 12th Symposium, 1981

6. Brundle, C. R., and Broughton, J. Q., "The Initial Interaction of Oxygen With Well-Defined Transition Metal Surfaces," in Chem. Physics of Solid Surfaces and Heterogeneous Catalysis, Elsevier, Amsterdam, vol. 3, Part A, p. 131-388 (1990)

7. Riley, J. A., and Giese, C. F., Interaction of Atomic Oxygen With Various Surfaces, J. Chem. Phys., 53, 1970, pp. 146-152.

8. Wood, B. J., “The Rate and Mechanism of Interaction of Oxygen Atoms ...," J. Phys. Chem., 75, 1971, pp. $2186-2193$.

9. Moe, K., and Moe, M. M., “The Effect of Adsorption on Densities.....”, Planet. Space Sci. 15, 1967, pp.1329-1332.

10. Moe, M. M. and Moe, K., "The Roles of Kinetic Theory and Gas-Surface Interactions in Measurements of UpperAtmospheric Density", Planet. Space Sci., 17, 1969, pp.917-922.

11. Moe, K., Moe, M. M., and Yelaca, N. W., "Effect of Surface Heterogeneity on the Adsorptive Behavior.... J. Geophys. Res, 77, 1972, pp. 4242-4247.

12. Hedin, A. E., Hinton, B. B., and Schmitt, G. A., "Role of Gas-Surface Interactions in the Reduction of OGO 6 Neutral Particle Mass Spectrometer Data," J. Geophys. Res., 78, 1973, pp. 4651-4668.

13. Offermann, D. and Grossmann, K. U., "Thermospheric Density and Composition As Determined By a Mass Spectrometer With Cryo Ion Source,” J. Geophys. Res., 78, 1973, pp. 8296-8304.

14. Gregory, J. C. and Peters, P. N., "A Measurement of the Angular Distribution of 5 Ev Atomic Oxygen Scattered off a Solid Surface in Earth Orbit, in Rarefied Gas Dynamics," Proceedings of 15th Symposium, Vol. 2, B. G. Teubner, Stuttgart, Germany, 1987, pp. 644-654.

15. Levine, A. S. (Ed.), LDEF - "69 Months in Space, First Post-Retrieval Symposium," NASA Conference Publication 3134 , (1991).

16. Murr, L. E., and Kinard, W. H., "Effects of Low-Earth orbit," American Scientist, 81, 1993, pp. 152-165.

17. Lake, L. R., and Nier, A. O., "Loss of Atomic Oxygen in Mass Spectrometer Ion Sources," J. Geophys. Res., $78,1973$.

18. Horowitz, R., and LaGow, H. E., J. Geophys. Res., 62, 1057, pp. 57-78.

19. Newton, G. P., Pelz, D. T., Miller, G. E., and Horowitz, R., "Response of Modified Gauges Aboard Explorer 17,” NASA TN D-2146 (1964). 
20. Mauersberger, K., Kayser, D. C., Potter, W. E., and Nier, A. O., "Seasonal Variation of Neutral thermospheric Constituents in the Northern Hemisphere," J. Geophys. Res., 81, 1976, pp. 7-11.

21. Yee, J. H., Abreu, V. J, and Dalgarno, A., "The Atmosphere Explorer Optical Glow Near Perigee Altitudes," Geophysical Res. Let., 12, 1985, pp. 651-654.

22. Sentman, L. H., "Free Molecule Flow Theory ...," Technical Report LMSC-448514, Lockheed Aircraft Corp., Sunnyvale, CA, (1961).

23. Schamberg, R., “A New Analytic Representation of Surface Interaction for Hyperthermal Free Molecular Flow,” RM-2313, the RAND Corp., Santa Monica, CA (1959).

24. Schamberg, R., "Analytic Representation of Surface Interaction...," in Aerodynamics of the Upper Atmosphere, R-339, the RAND Corp., Santa Monica, CA (1959).

25. Wolverton, R.W. (Ed.), "Flight Performance Handbook for Orbital Operations," Wiley, NY, pp. 2-336 to 2-359 (1963).

26. Izakov, M. N., "Some Problems of Investigating the Structure of the Upper Atmosphere and Constructing Its Models," Space Res. 5, 1965, p. 1191.

27. Cook, G. E., "Satellite Drag Coefficients,” Planet. Space Sci., 13, 1965, pp. 929-946.

28. Cook, G. E., "Drag Coefficients of Spherical Satellites,” Ann. Geophys., 22, 1966, pp. 53-64.

29. Moe, K., "Absolute Atmospheric Densities Determined From the Spin and Orbital Decays of Explorer 6." Planet. Space Sci., 14, 1966, pp. 1065-1075.

30. Karr, G. R., "A Study of Effects of Gas-Surface Interaction on Spinning Convex Bodies With Application To Satellite Experiments.” Rep. R-435. Coord. Sci. Lab., U. of Illinois, Urbana (1969).

31. Moe, K., "Recent Experimental Evidence Bearing on Satellite Drag Coefficients", AIAA J. 6, 1968, pp. $1375-1377$.

32. Beletsky, V. V., "An Estimate of the Character of the Interaction Between the Airstream and a Satellite," Kosmecheskie Issledovania 8 (2), 1970 (in Russia) 206-217.

33. Ching, B. K., Hickman, D. R., and Straus, J. M., "Effects of Atmospheric Winds and Aerodynamic Lift on the Inclination of the Orbit of the S3-1 Satellite," J. Geophys. Res., 82, 1977, pp. 1474-1480.

34. Imbro, D. R., Moe, M. M., and Moe, K., "On Fundamental Problems in the Deduction of Atmospheric Densities From Satellite Drag,” J. Geophys. Res., 80, 1975, pp. 3077-3086.

35. Sentman, L. H., "Comparison of the Exact and Approximate Methods For Predicting Free-Molecular Aerodynamic Coefficients,” Amer Rocket Soc. J., 31, 1961, pp. 1576-1579.

36. Moe, M. M., Wallace, S. D., and Moe, K., "Refinements in Determining Satellite Drag Coefficients: Method For Resolving Density Discrepancies," J of Guidance, Control and Dynamics, 16, 1993, pp. 441-445.

37. Moe, M. M., Wallace, S. D., and Moe, K., "Recommended Drag Coefficients For Aeronomic Satellites. The Upper Mesosphere and Lower thermosphere: a Review of Experiment and theory." Geophysical Monograph 87. American Geophysical Union, Washington, D. C, 1995, pp. 349-356.

38. DeVries, L., Personal communication (1971).

39. Moe, K., Moe, M. M., and Wallace, S. D., "Improved Satellite Drag Coefficient Calculations From Orbital Measurements of Energy Accommodation,” J. Spacecraft Rockets, 35, 1998, pp. 266-272.

40. Thomas, L. B., "A Collection of Some Controlled Surface thermal Accommodation Coefficient Measurements," in Rarefied Gas Dynamics, Proceedings of 5th Symposium, Academic Press, NY (1967).

41. Stickney, R. E., and Hurlbut, F. C., in "Rarefied Gas Dynamics," Proceedings, 3rd Symposium, Academic press, NY (1963).

42. Kostoff, R. N., Anderson, J. B., and Fenn, J. B., pp. 512-521 in Ref. 4.

43. Cress, G. H., Potter, A. E., Settecerri, T. J., Sherrill, G. P., and Talent, D. L., "Radar and Optical Ground Measurements Final Report......," NASA Report JSC -27242, NASA Johnson Space Center, Houston, TX (1996).

44. Moe, K., and Bowman, B. R., "Effects of Surface Composition and Treatment on Drag Coefficients of Spherical Satellites," AAS Paper 05-258 (2005), AAS Publications Office, San Diego, CA.

45. Tan, A. and Badhwar, G. D., “Analysis of Orbital Decay of the ODERACS Spheres,” Appendix A of Ref. 43.

46. Pardini, C., Anselmo, L., Moe, K., and Moe, M. M., "Drag and Energy Accommodation Coefficients during Sunspot Maximum," Adv. Space Res. 45, pp. 638-650 (2010).

47. Vashchenkov, P. V., Kashnovsky, A. V., and Ivanov, M. S., "High-Altitude Aerodynamics of the Clipper Spacecraft", CD 15 in Appendix to Rarefied Gas Dynamics, 26th International Symposium (2008).

48. Fujita, K, and Noda, A., "Astrodynamics of Satellites on a Super Low-Earth Orbit”, pp. 772-777, Rarefied Gas Dynamics, 26th International Symposium (2008).

49. Pilinski, M. D., Argrow, B. M., and Palo, S. E., “A Semi-Empirical Model ...,” J. Spacecraft Rockets, in press, 2010.

50. Emmert, J. T., Meier, R. R., Picone, J. M., Lean, J. L., Christensen, A. B., “Thermospheric Density, 2002-2004: Timed/GUVI Dayside Limb Observations and Satellite Drag,” J. Geophys. Res. - Part A-Space Physics 111, nA10, p 11, 1 Oct. 2006 , pp.1.

51. Bruinsma, S., Tamagnan, D., and Biancale, R., Planet. Space Sci., 52, 2004, pp. 297-312.

52. Marcos, F. A.,"New Measurements of Neutral Thermospheric Density..." Paper AAS 05-251, Am. Astronaut. Soc. 2005.

53. Bowman, B. R., Marcos, F. A., Moe, K., and Moe, M. M., "Determination of Drag Coefficient Values for CHAMP and GRACE Satellites Using Orbit Drag Analysis," Paper AAS 07-259, American Astronautical Society, San Diego, CA. (2007).

54. Doornbos, E., Foerster, M., Fritsche, B., van Helleputte, T., v. d. Ijssel, J., Koppenwallner, G., Luehr, H., Rees, D., Visser, P., and Kern, M., “Air Density Models Derived from Multi-Satellite....," Tech. Rep. DEOS/TU Delft, Netherlands (2009).

55. Moe, K., Moe, M. M., and Doornbos, E., "Outstanding Issues Related to Thermospheric Measurements and Modeling", to be presented at the COSPAR meeting, Bremen, July, 2010. 